\title{
COVID-19 IN PATIENTS WITH CHRONIC MYELOID LEUKEMIA: MANAGEMENT CHALLENGES AND OUTCOMES
}

\author{
Vasile MUSTEATA ${ }^{1,2}$ 凹 \\ ${ }^{1}$ Discipline of Hematology, "Nicolae Testemitanu" State University of Medicine and Pharmacy, Chisinau, \\ Republic of Moldova \\ ${ }^{2}$ Department of Hematology, Institute of Oncology, Chisinau, Republic of Moldova \\ Received 11 Oct 2021, Accepted 04 Nov 2021 \\ https://doi.org/10.31688/ABMU.2021.56.4.09
}

\begin{abstract}
Introduction. A growing number of studies indicates the increased susceptibility of patients with hematologic malignancies to severe acute respiratory syndrome coronavirus 2 (SARS-CoV-2) infection as compared to the apparently healthy population.

The objective of our study was to identify and evaluate the contemporary epidemiological and diagnosis patterns of chronic myeloid leukemia (CML) in the context of coronavirus disease 2019 (COVID-19) pandemic, to optimize the management of this hematologic malignancy.
\end{abstract}

Material and methods. This analytical, descriptive and prospective study included 15 patients with CML and COVID-19, who were treated and followed up in the Institute of Oncology Chisinau, Republic of Moldova, between 2005-2021. SARS-CoV-2 infection was diagnosed in these patients between April 2020 September 2021.

Results. These 15 cases represented $10.6 \%$ out of 142 patients diagnosed with CML and followed up under the treatment with tyrosine kinase inhibitors. There were $9(60.0 \%)$ males and $6(40.0 \%)$ females, with the

\section{Résumé}

COVID-19 chez les patients atteints de leucémie myéloïde chronique: défis de gestion et résultats

Introduction. Le nombre accru d'études indique une sensibilité des patients atteints d'une tumeur hématologique maligne à l'infection par le SRAS-CoV-2 par rapport à la population apparemment en bonne santé. Objectifs. Identification et évaluation des profils épidémiologique et diagnostique contemporains de la leucémie myéloïde chronique (LMC) dans le contexte de la pandémie de la maladie du virus Corona 2019 (COVID-19), afin d'optimiser la prise en charge de cette tumeur maligne hématologique.

Matériel et méthodes. Cette étude analytique, descriptive et prospective a inclus 15 patients atteints de LMC et COVID-19, qui ont été traités et suivis à l'Institut d'oncologie de Chisinau, en République de Moldova, entre 2005 et 2021. L'infection au SRAS-CoV-2 a été diagnostiquée chez ces patients entre avril 2020 et septembre 2021.

Résultats. Ces 15 cas représentaient $10,6 \%$ des 142 patients diagnostiqués avec une LMC et suivis sous 
male/female ratio 1.5/1. The patients' age at diagnosis of COVID-19 ranged between 38-74 years (average age 54.2 years). According to the World Health Organization classification of COVID-19 severity, mild forms were registered in $4(26.7 \%)$ cases, moderate in $6(40.0 \%)$ and severe in $5(33.3 \%)$ cases. Eight (53.3\%) patients were hospitalized for treatment of COVID-19: 5 patients with severe forms and 3 patients with moderate forms. Viral pneumonia was diagnosed in 6 patients: 5 with severe and one with moderate form of COVID-19. Non-infectious comorbidities were registered in 4 of 5 patients with severe COVID-19 forms.

Conclusions. COVID-19 commonly affects CML patients of male gender and older than the average age of patients with CML. Patients without hematologic response presented an unfavorable evolution of both diseases, CML and COVID-19.

Keywords: chronic myeloid leukemia, COVID-19, age, comorbidities.

\section{INTRODUCTION}

Chronic myeloid leukemia (CML) is a clonal neoplastic pathology of hematopoietic system, which results from the malignant transformation of the stem cell, maintaining the ability of differentiation to the all cell lines, especially myeloid lineage ${ }^{1-5}$. CML is characterized by the uncontrolled multiplication of myeloid series cells, with the increased circulating and total granulocyte pool. It represents $15-20 \%$ of all leukemia cases in adults, being the most common chronic myeloproliferative neoplasm ${ }^{6-9}$. CML morbidity increases with age, with the highest incidence between 45 and 60 years ${ }^{6,10}$. A growing number of publications suggests the increased susceptibility of patients with malignant neoplasms to severe acute respiratory syndrome coronavirus 2 (SARS-CoV-2) infection and its increased incidence (1\%) as compared to the apparently healthy population $(0.1 \%)$, but these estimations are controversial, and the infection risk is not determined according to the type of hematological malignancy ${ }^{11-13}$. The increasing incidence in the working-age population, commonly delayed diagnosis of CML and the significant level of disability-adjusted life years rate ${ }^{14}$ in the advanced phases of the disease can be considered as major issues of public health and hematooncology, justifying the study of its traitement par inhibiteurs de la tyrosine kinase. Il y avait $9(60,0 \%)$ hommes et $6(40,0 \%)$ femmes, avec un rapport hommes/femmes de 1,5:1. L'âge des patients au moment du diagnostic de COVID-19 variait entre 38 et 74 ans (âge moyen 54,2 ans). Selon la classification de l'Organisation mondiale de la santé de la gravité du COVID-19, des formes bénignes ont été enregistrées dans $4(26,7 \%)$ cas, modérées dans $6(40,0 \%)$ et sévères dans $5(33,3 \%)$ cas. Huit $(53,3 \%)$ patients ont été hospitalisés pour le traitement du COVID-19, 5 patients avec des formes sévères et 3 patients avec des formes modérées. Une pneumonie virale a été diagnostiquée chez 6 patients, 5 avec des formes sévères et un avec une forme modérée de COVID-19. Des comorbidités non infectieuses ont été enregistrées chez 4 des 5 patients présentant une forme sévère de COVID-19. Conclusions. COVID-19 affecte couramment les patients atteints de LMC de sexe masculin et plus âgés que l'âge moyen des patients atteints de LMC. Les patients sans réponse hématologique ont présenté une évolution défavorable des deux maladies, LMC et COVID-19.

Mots-clés: leucémie myéloïde chronique, COVID-19, âge, comorbidités.

epidemiological and diagnosis features in the current epidemiological context.

The OBJECTIVE OF THE STUDY was the identification and evaluation of the contemporary epidemiological and diagnosis patterns of CML in the context of COVID-19 pandemic, to optimize the management of this hematologic malignancy.

\section{Material AND MEthods}

This analytical, descriptive and prospective study included 15 patients with CML and COVID-19, who were treated and followed up in the Institute of Oncology Chisinau, Republic of Moldova, between 2005-2021. SARS-CoV-2 infection was diagnosed between April 2020-September 2021 only in these 15 patients out of the whole group of 142 patients with CML treated with tyrosine kinase inhibitors (TKIs) at the Institute of Oncology.

The type of leukemia was diagnosed according to the Revised 2017/2020 World Health Organization (WHO) Classification of Tumours of Hematopoietic and Lymphoid Tissues ${ }^{15-17}$. All CML cases were diagnosed in chronic phase by the molecular tests of the bone marrow (BM) and peripheral blood (PB). The quantitative reverse transcription polymerase chain reaction (RT-PCR) was accomplished with the aim to 
determine the expression of the BCR-ABL p210 and p190 transcripts. Five transcription products (b2a2, $\mathrm{b} 3 \mathrm{a} 2, \mathrm{~b} 2 \mathrm{a} 3, \mathrm{~b} 3 \mathrm{a} 3$ si e1a2) were analysed by the usage of the quantitative PCR test. Nucleic acid amplification testing with a RT-PCR assay was performed to detect SARS-CoV-2 RNA from the upper respiratory tract, as the preferred initial diagnostic test for COVID-19. The severity of COVID-19 was determined according to the WHO Guidelines for certification and classification (coding) of COVID-1918,19. The prospective practical importance of the study consists in assessing the risk and patterns of infection with SARS-CoV-2 in CML patients and reducing the negative effects of the COVID-19 pandemic on the management of CML.

\section{Results}

These 15 patients with CML and SARS-CoV-2 infection represented $10.6 \%$ of 142 patients diagnosed with CML and currently followed up under the treatment with TKIs in the Institute of Oncology. Of these 15 cases, COVID-19 occurred only in 2 (5.9\%) of 34 patients primarily diagnosed with CML and treated with TKIs since the onset of COVID-19 pandemic in the Republic of Moldova (07.03.2021) until the last COVID-19 case before finalizing this study (30.09.2021). Thus, the patients diagnosed with CML before the onset of COVID-19 pandemic had a higher morbidity rate of SARS-CoV-2 infection as compared to patients with shorter duration of CML $(12.0 \%$ versus $5.9 \%)$. There were 9 males and 6 females, with the male/female ratio 1.5/1 (Table 1). In our study,
SARS-CoV-2 infection affected mostly males. The patients' age at the diagnosis of COVID-19 ranged between 38-74 years (average age 54.2 years). Seven (46.7\%) patients were under 50 years old, $8(53.3 \%)$ patients above 50 years, and 6 of them above 60 years old. Only 2 (13.3\%) patients were under 40 years old. These data suggest that the older CML patients are more susceptible to COVID-19.

According to the WHO Classification of COVID-19 severity, mild forms were registered in 4 (26.7\%) cases, moderate in $6(40.0 \%)$ cases and severe in $5(33.3 \%)$ cases $^{18,19,21}$. Three patients with severe forms and two patients with moderate forms were aged between $60-70$ years old.

The duration of the hematologic malignancy until the diagnosis of SARS-CoV-2 infection varied between 46-214 months (the average period 112 months). No correlation was found between the duration of the CML and the severity of COVID-19. None of the patients was vaccinated against SARS-CoV-2 before the disease.

The Eastern Cooperative Oncology Group (ECOG) score of performance status of CML patients ranged between $0-2$ at the time of diagnosis of SARS-CoV-2 infection. The ECOG score was 2 only in $2(13.3 \%)$ patients, who experienced severe COVID-19.

This study evaluated the impact of Sokal score on COVID-19 morbidity in CML patients. SARS-CoV-2 affected 8 (53.3\%) low-risk patients, 5 (33.3\%) intermediate-risk and 2 (13.4\%) high-risk patients. Moderate and severe COVID-19 forms developed in 5 (33.3\%) low-risk patients. Five (33.3\%)

Table 1. Clinical and biological characteristics of CML cases according to the severity of COVID-19.

\begin{tabular}{|c|c|c|c|c|c|c|c|c|c|c|c|c|c|c|c|}
\hline \multirow{2}{*}{$\begin{array}{c}\text { Category of } \\
\text { COVID-19 } \\
\text { infection }\end{array}$} & \multirow{2}{*}{$\begin{array}{l}\text { Number of } \\
\text { patients } \\
\text { percentage }\end{array}$} & \multicolumn{3}{|c|}{ CML phase } & \multirow{2}{*}{ 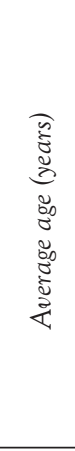 } & \multirow{2}{*}{ 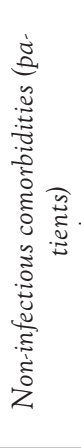 } & \multicolumn{2}{|c|}{ Gender } & \multicolumn{2}{|c|}{$\begin{array}{l}\text { Complete } \\
\text { hematologic } \\
\text { response }\end{array}$} & \multicolumn{3}{|c|}{$\begin{array}{l}\text { Risk group } \\
\text { according to } \\
\text { Sokal score } \\
\text { (patients) }\end{array}$} & \multirow{2}{*}{ 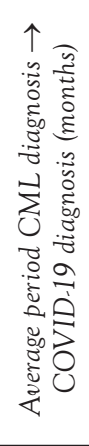 } & \multirow{2}{*}{ 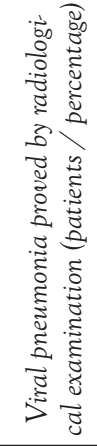 } \\
\hline & & 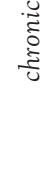 & $\frac{\vec{\Xi}}{\stackrel{\vec{J}}{ت}}$ & 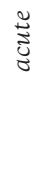 & & & $\frac{\ddot{\Xi}}{\tilde{\Xi}}$ & 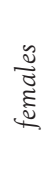 & 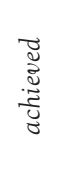 & 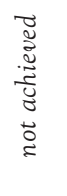 & క & 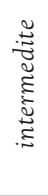 & $\stackrel{\overrightarrow{0}}{\stackrel{\vec{\Xi}}{\Xi}}$ & & \\
\hline Mild & $4 / 26.7$ & 4 & - & - & 53.3 & - & 2 & 2 & 4 & - & 3 & - & 1 & 133 & - \\
\hline Moderate & $6 / 40.0$ & 6 & - & - & 50.0 & - & 4 & 2 & 6 & - & 4 & 2 & - & 82.3 & $1 / 6.7$ \\
\hline Severe & $5 / 33 / 3$ & 3 & 1 & 1 & 57.0 & 4 & 3 & 2 & 3 & 2 & 1 & 3 & 1 & 130.9 & $5 / 33.3$ \\
\hline Total & $15 / 100$ & 13 & 1 & 1 & 54.2 & 4 & 9 & 6 & 13 & 2 & 8 & 5 & 2 & 112 & $6 / 40$ \\
\hline
\end{tabular}

Legend: $\mathrm{CML}=$ chronic myeloid leukemia, COVID-19 = Coronavirus Disease 2019 
intermediate-risk patients and one (6.7\%) high-risk patient also suffered from moderate and severe forms of COVID-19. One (6.7\%) high-risk patient experienced a mild form of COVID-19. The intermediate and high Sokal score, thus, did not influence the rate and severity of COVID-19 in CML patients.

The infection with SARS-CoV-2 occurred under the treatment with imatinib in 9 (60.0\%) cases, nilotinib in $4(26.7 \%)$ and under the treatment with ponatinib in $2(13.3 \%)$ cases. Nevertheless, the shares of these cases within the totalities of CML patients treated with imatinib $(\mathrm{N}=62)$, nilotinib $(\mathrm{N}=29)$ and ponatinib $(\mathrm{N}=15)$ were $14.2 \%, 13.8 \%$ and $13.3 \%$, respectively. No significant difference of COVID-19 morbidity was registered between patients treated with different generations of TKIs.

COVID-19 developed in 13 (86.7\%) patients with complete hematologic response and in $2(13.3 \%)$ patients without stable response to treatment with TKIs.

Eight $(53.3 \%)$ patients were hospitalized for treatment of COVID-19, including all $5(33.3 \%)$ with severe and 3 (20.0\%) with moderate forms. Viral pneumonia was diagnosed by radiological examinations in $6(40.0 \%)$ patients, including $5(33.3 \%)$ with severe and one (6.7\%) with moderate COVID-19. Seven (46.7\%) patients were discharged from the hospital with a recovery from COVID-19. One (6.7\%) patient with severe COVID-19 form died because of the progression of CML into the acceleration phase and SARS-CoV-2 infection consequences. One (6.7\%) patient with severe evolution of COVID-19 died because of bilateral viral pneumonia, acute cardio-respiratory failure and CML progression into an acute phase (myeloid blast crisis). No other clinico-hematologic relapses and progression of CML were registered. These results allowed to assume that those patients without hematologic response experienced the unfavorable evolution of both diseases: CML and COVID-19.

Non-infectious comorbidities were registered in 4 of 5 patients with severe COVID-19 evolution, especially cardio-vascular diseases in 3 patients and type 2 diabetes mellitus in 2 patients.

The moderate COVID-19 occurred repeatedly only in one (7.7\%) alive patient with the initially mild form, stable complete hematologic response and minor molecular relapse of CML. The repeated SARS-CoV-2 infection evolved without pneumonia and did not require hospitalization.

\section{Discussion}

Some studies regarding the epidemiological and diagnostic features of CML have already been published in the context of the pandemic with
COVID-19. We searched the medical literature using Google Search, PubMed, Z-library, NCIB, Medscape, Hinari database, by the keywords: "chronic myeloid leukemia", "COVID-19", "diagnosis", "incidence", "morbidity rate", "prevalence", "mortality", "survival", "management". More than 50 bibliographic sources have been studied. Thirty-two relevant primary sources were identified and selected on the basis of a significant impact score and scientific, reproducible and transparent approach, followed by the consequent data extraction and analysis. Intending to minimize errors, a copy of the data extraction sheet was initially produced, listing all the elements to be selected from the primary studies ${ }^{20}$.

The epidemiological studies indicate the occurrence of CML at any age, with an average age of 50 years at the time of diagnosis. Approximately $2.5 \%$ of cases with CML are attributed to the age group under 20 years, $7.4 \%$ to the age group between $20-34$ years and $33 \%$ to the age group under 40 years ${ }^{6,10,22-25}$. CML predominantly affects males, the male/female ratio being $1.2-1.3 / 1^{26,27}$.

In the current epidemiological context, the association of COVID-19 with CML is of both scientific and practical interest, taking into account the immunocompromised status of patients with this myeloproliferative neoplasm. The epidemiological and clinical aspects of COVID-19 in patients with CML are controversially reflected in the literature ${ }^{28}$.

In this study, the average age of CML patients with COVID-19 (54.2 years) was higher, as compared with the totality of CML patients. COVID-19 affected mostly the males with CML, with the male/female ratio of 1.5/1.

The rate of moderate and severe COVID-19 proved to be higher in CML patients than in the general population ${ }^{29,30}$. The CANDID multicenter study reported to International Chronic Myeloid Leukemia Foundation (iCMLf) 110 cases of CML from 20 countries, especially from Europe - 61\%, Asia 15\%, South America - 12\%, North America - 10\%, Africa - 2\% and Oceania - 1\%. At the beginning of COVID-19 pandemic, 46 hematologists reported only $91(0.7 \%)$ cases of COVID-19 in 12,236 patients with CML, which might suggest that the immune status in the chronic phase of CML was not a contributing factor in the development of this viral disease ${ }^{28}$. The age of patients diagnosed with COVID-19 ranged from 18 to 89 years, with a mean age of 54 years, which exceeded the mean age in the total CML population reported in the majority of studies, ${ }^{5,6,31}$ and was approaching to the same age indicator in our study. Nevertheless, the mean time from confirmation of CML diagnosis to the development of COVID-19 was shorter than in our patients (84 months), with 
the limits ranging between $6-300$ months. The study of the infected patients showed that $77(70 \%)$ received standard chemotherapy with TKIs, 33 (30\%) discontinued it during the diagnosis and treatment of the viral infection ${ }^{28}$. Eighteen (16\%) patients were not treated with chemotherapy because of different reasons. COVID-19 progressed asymptomatically in 8 (7\%) cases, in mild forms without hospitalization in $49(45 \%)$ cases, moderate forms with hospitalization in $19(17 \%)$ cases, severe forms with treatment in intensive care units in 19 (17\%) cases. The rate of mortality from COVID-19 was $10.9 \%$. The survival was analysed according to sex, age, chemotherapy protocol, comorbidities, anti-COVID-19 treatment and level of economic development of the country. The univariate analysis revealed a higher mortality rate in patients aged $\geq 75$ years $(60 \%$ vs $7 \%$ in those under 75 years, $p<0.001$ ), with severe forms of infection $(63 \%$ vs $0 \%$ in non-severe forms, $p<0.001)$ and in patients over 75 years old treated with first generation TKIs ( $25 \%$ vs $3 \%$ against the background of second generation TKIs vs $0 \%$ in those without TKIs therapy, $\mathrm{p}=0.003$ ).

Another study of 125 hospitalized patients with hematologic tumours indicated a 10 percentile rate of COVID-19, but none of the subjects was diagnosed with $\mathrm{CML}^{12}$. Nonetheless, in the same time of pandemic with SARS-CoV-2 infection, 530 cases of CML were studied in 29 medical centers of the Anti-Cancer Association of Hubei (China) ${ }^{32}$. Five patients developed COVID-19, so the rate of this disease was $0.9 \%$, nine times exceeding the rate $(0.1 \%)$ in the healthy population, but lower than $10 \%$ reported in the hospitalized patients with other malignant hematological tumours, and $7 \%$ in the healthy health care providers. The evolution of the infectious process in CML cases has proved to be typical both in terms of the clinical picture and imaging of the chest on computed tomography scan. As in our study, the co-variables associated with an increased risk of developing COVID-19 among patients with CML were the exposure to persons infected with SARS-CoV-2 $(\mathrm{p}=0.037)$, lack of complete hematologic response ( $\mathrm{p}$ $=0.003)$ and comorbidities $(p=0.024)$. A higher risk of developing COVID-19 was observed in patients with advanced CML ( $\mathrm{p}=0.004)$, despite obtaining a complete cytogenetic response or major molecular response in the period of exposure to SARS-CoV-2. During the treatment with TKIs, COVID-19 occurred in one of 21 patients under the third generation medication, in 3 of 346 under the imatinib medication, and in none of the 162 who received the second generation $(p=0.096)$. These data may suggest that the first and second generations of TKIs are associated with a reduced risk of COVID-19, which doesn't match the corresponding data from the previous study. Other co-variables, such as age and duration of treatment with TKIs, were not associated with an increased risk of developing COVID-19. It may be suggested that patients with identified risk factors should benefit from the increased surveillance in terms of SARS-CoV-2 infection, with the possibility of protective isolation and repeated testing until obtaining the complete hematologic response.

The main limitations of this study are related to the small number of CML patients diagnosed with SARS-CoV-2 infection.

\section{Conclusions}

This study indicated the predominance of COVID-19 in the working-age patients with CML, due to the immunocompromised status and comorbidities. COVID-19 frequently affects unvaccinated CML patients of male gender and older than the average age of CML patients. SARS-CoV-2 infection is encountered in CML patients more frequently than in the general population, but less commonly than in other hematologic malignancies, being not influenced by Sokal score in our study.

Patients without hematologic response presented an unfavourable evolution of both diseases: CML and COVID-19. The mortality rate in patients with CML associated with COVID-19 is higher in those $\geq 75$ years old, with the advanced CML stage, severe forms of infection and in persons over 75 years old treated with first generation TKIs. No significant difference of COVID-19 morbidity was registered in patients below 75 years old, treated with different generations of TKIs.

The management of intermediate and high-risk CML patients should comprise the careful monitoring for SARS-CoV-2 infection, with protective isolation and repeated testing for COVID-19 until obtaining the complete hematologic response.

\section{Compliance with Ethics Requirements:}

"The author declares no conflict of interest regarding this article"

"The author declares that all the procedures and experiments of this study respect the ethical standards in the Helsinki Declaration of 1975, as revised in 2008(5), as well as the national law. Informed consent was obtained from all the patients included in the study. The research protocol No. 9 was approved on September 21, 2015 by the Research Ethics Board of the "Nicolae Testemitanu" State University of Medicine and Pharmacy, Chisinau, Republic of Moldova."

"No funding for this study" 


\section{Acknowlegements}

"This study was supported by the "Nicolae Testemitanu" State University of Medicine and Pharmacy and Institute of Oncology, Chisinau, Republic of Moldova. It was approved by the University Senate and Scientific Council of the Institute of Oncology. Reverse transcription polymerase chain reaction was performed in the Laboratory of immunology and molecular genetics of the Institute of Oncology."

\section{References}

1. Baccarani M, Deininger M, Rosti G,et al. European Leukemia Net recommendations for the management of chronic myeloid leukemia: 2013. Blood 2013; 122: 872-884.

2. Cortes EJ, List A, Kantarjian H. Chronic myelogenous leukemia. In: Pazdur R, Coia LR, Hoskins WJ, et al. Cancer Management: A Multidisciplinary Approach, 8th ed. New York, CMP Healthcare Media, 2004, pp. 773-786.

3. Cortes JE, Kantarjian H. How I treat newly diagnosed chronic phase CML. Blood 2012; 120 (7):1390-1397.

4. Hehlmann R. Chronic myeloid leukemia, 2nd ed. Springer Nature Switzerland AG, 2021: 273 p.

5. Thompson PA, Kantarjian HM, Cortes JE. Diagnosis and treatment of chronic myeloid leukemia in 2015. Mayo Clinic Proceedings 2015; 90(10):1440-1454.

6. Buyukasik YC, Haznedaroglu IC, Ilhan O. Chronic myeloid leukemia: practical issues in diagnosis, treatment and follow-up. International Journal of Hematology and Oncology, 2010; 20 (2), Suppl. 1: 1-12.

7. Al-Achkar W, Moassass F, Youssef N, Wafa A. Correlation of p210 BCR-ABL transcript variants with clinical, parameters and disease outcome in 45 chronic myeloid leukemia patients. Journal of BUON 2016;21(2):444-449.

8. Jabbour E, Kantarjian H. Chronic myeloid leukemia: 2018 update on diagnosis, therapy and monitoring. Am J Hematol. 2018; 93: 442-459.

9. Quintas-Cardama A, Cortes JE. Chronic myeloid leukemia: diagnosis and treatment. Mayo Clin Proc 2006; 81(7):973-988

10. Dorfman LE, FlorianiMA, Oliveira TM,et al.The role of cytogenetics and molecular biology in the diagnosis, treatment and monitoring of patients with chronic myeloid leukemia $J$ Bras. Patol. Med. Lab. 2018;54 (2):83-91.

11. Kutikov A, Weinberg DS, Edelman MJ, et al. A war on two fronts: cancer care in the time of COVID-19. Ann Intern Med. 2020;172(11):756-758.

12. He W, Chen L, Yuan G, et al. COVID-19 in persons with hematological cancers. Leukemia 2020;34 (6):1637-1645.

13. Liang W, Guan W, Chen R,et al. Cancer patients in SARS-CoV-2 infection: a nationwide analysis in China. Lancet Oncol. 2020;21:335-7.

14. Fitzmaurice C, Akinyemiju TF, Al Lami FH,et al. Global, regional, and national cancer incidence, mortality, years of life lost, years lived with disability, and disability-adjusted life-years for 29 cancer groups, 1990 to 2016. A systematic analysis for the Global Burden of Disease Study. JAMA Oncol. 2018;4(11):1553-1568.
15. Swerdlow SH, Campo E, Harris NL, et al. WHO Classification of Tumours of Haematopoietic and Lymphoid Tissues, Revised 4th ed. IARC: Lyon, France, 2017.

16. ESMO Guidelines Committee. Chronic myeloid leukemia: ESMO Clinical Practice Guidelines for diagnosis, treatment and follow-up. Annals of Oncology 2017;28(4): iv41-iv51.

17. Carbone A. Classification of tumors of the hematopoietic and lymphoid tissues. Discovering diseases - defining their features. Bloods 2020;1:7-9.

18. World Health Organisation. International guidelines for certification and classification (coding) of COVID-19 as cause of death. Based on ICD International Statistical Classification of Diseases. WHO/HQ/DDI/DNA/CAT, 2020, $14 \mathrm{p}$

19. National Institutes of Health. COVID-19 Treatment Guidelines Panel. Coronavirus Disease 2019 (COVID-19) Treatment Guidelines. Available athttps://www.covid19treatmentguidelines.nih.gov/(accessed 10 Oct, 2021)

20. Tintiuc D, Badan V, Raevschi E, et al. Biostatistica si metodologia cercetarii stiintifice. Chisinau: CEP Medicina, 2011, $344 \mathrm{p}$.

21. Xiong F, Wang Y, You T, et al. The clinical classification of patients with COVID-19 pneumonia was predicted by Radiomics using chest CT. Medicine 2021;100(12):1-8.

22. Musteata V. Good clinical practice in chronic myeloid leukemia: advances and prospects at the Institute of Oncology of Moldova. Journal of BUON 2010;15:188-189.

23. Turkina AG, Zaritskii Au, Shuvaev VA, et al.Clinical recommendations for the diagnosis and treatment of chronic myeloid leukemia. Clinical Oncohematology 2017;10(3):294-316.

24. Cancer.Net Editorial Board. Leukemia - Chronic Myeloid CML: Statistics. https://www.cancer.net/cancer-types/ leukemia-chronic-myeloid-cml. 2018.

25. Musteata V, Stratan V, Catrinici L,et al. Management of elderly patients with chronic myeloproliferative hemopathies. The Moldovian Medical Journal 2020;63(6):12-15.

26. Berger U, Maywald O, Pfirrmann M, et al. Gender aspects in chronic myeloid leukemia: long-term results from randomized studies. Leukemia 2005;19:984-9.

27. Höglund M, Sandin F, Simonsson B. Epidemiology of chronic myeloid leukaemia: an update. Ann Hematol. 2015;94 (Suppl 2):S241-247.

28. Rea D, Mauro MJ, Cortes JE, et al. COVID-19 in Patients with Chronic Myeloid Leukemia (CML): Results from the International CML Foundation (icmlf) CML and COVID-19 (CANDID) Study. Blood 2020;136 (Suppl 1): 46-47.

29. Verity R, Okell L, Dorigatti I, et al. Estimates of the severity of coronavirus disease 2019: a model-based analysis. The Lancet. 2020;20(6):669-677.

30. Kabir MT, Uddin MS, Hossain MF, et al. nCOVID-19 pandemic from molecular pathogenesis to potential investigational therapeutics. Frontiers in Cell and Developmental Biology 2020; 8:616.

31. Musteata V. Clinico-laboratory pertinences and management of relapsed and refractory chronic myeloid leukemia. Journal of BUON 2021;26(3):1165-1168.

32. Weiming L, Danyu W,Jingming G, et al. COVID-19 in persons with chronic myeloid leukemia. Leukemia 2020;34:1799-1804. 